\title{
Health-care providers' perspectives on uncertainty generated by variant forms of newborn screening targets
}

\author{
Paul J. Azzopardi, MSc (1) ${ }^{1}$, Ross E. G. Upshur, MD², Stephanie Luca, MA ${ }^{3}$, Viji Venkataramanan, MA $^{3}$, \\ Beth K. Potter, PhD ${ }^{4}$, Pranesh K. Chakraborty, $\mathrm{MD}^{5}$ and Robin Z. Hayeems, PhD ${ }^{1,3}$
}

\begin{abstract}
Purpose: Despite the public health successes of newborn bloodspot screening, uncertainty associated with variant forms of primary screening targets has led to discrepancies in medical management. This study explored health-care providers' approaches to managing atypical forms of inherited metabolic diseases (IMDs) in the absence of evidence-based guidelines.

Methods: Semistructured telephone interviews were conducted with metabolic specialists. 3-Methylcrotonyl CoA deficiency and variant forms of phenylketonuria, biotinidase deficiency, and fatty acid oxidation disorders were considered. Data were analyzed inductively and deductively using a novel taxonomy of uncertainty.

Results: Health-care providers $(n=12)$ navigate diagnostic, prognostic, and therapeutic challenges of uncertainty while interpreting patient and family attitudes, preferences, and ideas in the care of children with these result types. Participants explained the limits of classifying mild and atypical metabolic phenotypes.
\end{abstract}

Participants also described the challenge of finding balance between cautious care and overmedicalization. Developing consistent care plans and honest communication with families were perceived as effective strategies when navigating uncertainty.

Conclusion: Providers' experiences suggest a need for transparent and accessible guidelines that account for challenges associated with uncertainty generated by screening. Timely consideration of this challenge is warranted with increasing emergence of genotype-first approaches to screening.

Genetics in Medicine (2020) 22:566-573; https://doi.org/10.1038/s41436019-0670-3

Keywords: medical uncertainty; newborn screening; inherited metabolic disease; health-care providers; qualitative methods

\section{INTRODUCTION}

The US Centers for Disease Control and Prevention list declared newborn bloodspot screening (NBS) as one of the ten great public health achievements of the past decade. ${ }^{1}$ Future advances in NBS may integrate massively parallel sequencing as a first-tier test, potentially allowing for advances in the scalability, specificity, and customizability of screening programs. Increasingly, genotypic information is being integrated to optimize the precision of screening algorithms and to enable the identification of a broader range of screening and diagnostic targets in the newborn period. ${ }^{2,3}$ However, barriers to using first-tier genome sequencing in NBS include high cost of sequencing infrastructure, test sensitivity, interpretive and management complexity related to uncertain penetrance and mild and/or uncertain variants, and controversy with respect to how expansive screening panels ought to be. ${ }^{4}$ In light of these barriers, early targets for genotype-first approaches may be limited to diseases for which biochemical testing is impossible (i.e., biochemical biomarkers do not exist) or impractical (measurement of a biomarker is too costly or complex).

The current NBS landscape offers an opportunity to prepare for and better understand clinical practice concerns germane to using sequencing as a first-tier test. For example, current complexities associated with the interpretation and management of variant forms of primary screening targets foreshadow a key challenge associated with genotype-first NBS. ${ }^{5}$ This challenge is a current concern for inherited metabolic disease (IMD) specialists, who care for screen-positive infants. For instance, in Michigan, 142 children diagnosed with biotinidase deficiency (BIO) were followed over 25 years to find that 22 had profound enzyme deficiency and 120 had only partial deficiency. ${ }^{6}$ Infants with profound BIO are at risk of cognitive disability, hearing loss, and optic atrophy. ${ }^{6}$ Of critical importance, infants with partial BIO and other nonclassic disorders may be labeled, monitored, and treated with little certainty that they are at risk of ever becoming symptomatic. Persistent uncertainty commonly results from

\footnotetext{
${ }^{1}$ Institute of Health Policy, Management and Evaluation, University of Toronto, Toronto, ON, Canada; ${ }^{2}$ Dalla Lana Faculty of Public Health, University of Toronto, Toronto, ON, Canada; ${ }^{3}$ Child Health Evaluative Sciences, Hospital for Sick Children, Toronto, ON, Canada; ${ }^{4}$ Department of Epidemiology and Community Medicine, Faculty of Medicine, University of Ottawa, Ottawa, ON, Canada; ${ }^{5}$ Department of Pediatrics, Children’s Hospital of Eastern Ontario, Ottawa, ON, Canada. Correspondence: Robin Z. Hayeems (robin.hayeems@sickkids.ca)
} 


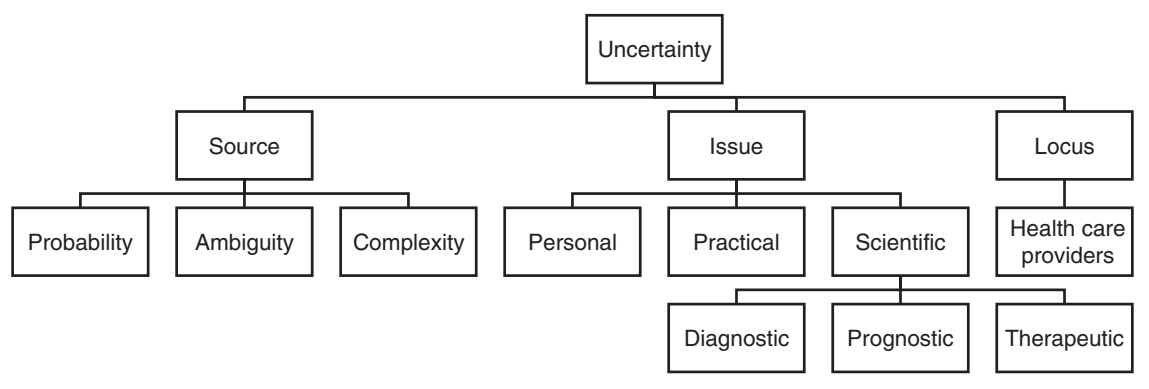

Fig. 1 The 3-dimensional taxonomy of uncertainty.

out-of-range laboratory findings. For example, a Taiwan study showed that 40 cases of mild hyperphenylalaninemia (MHP, $120-600 \mu \mathrm{M})$ were identified for 25 cases of classic phenylketonuria $(\mathrm{PKU},>600 \mu \mathrm{M}) .^{7}$ Uncertainty may also arise from variants associated with a nonclassic IMD form. Among 57 confirmed cases of medium-chain acyl-CoA dehydrogenase deficiency (MCADD), 9 infants were identified with a mild folding variant (c.199T $>$ C) of unknown clinical prognosis and 13 infants were identified with previously unknown variants. ${ }^{8}$ Nonclassic and partial forms of IMD tend to lack evidenceand consensus-based guidelines for navigating patient management. ${ }^{6,-12}$ Therefore, uncertainty can persist for months or years and is accompanied by inconsistent clinical interpretations and management strategies. ${ }^{13-15}$ With firsttier sequencing, medical uncertainty is likely to increase given the unknown penetrance of identified variants in the absence of symptoms and/or family history. ${ }^{16}$

Han and colleagues ${ }^{17}$ characterize uncertainty as the perceived lack of health-related knowledge, classified according to its sources, issues, and loci (Fig. 1). Sources of uncertainty include unpredictability, ambiguity, and complexity of medical information. Uncertainty can generate practical challenges for health-care providers, patients, laboratory scientists, and program leaders. While the literature articulates a range of complex feelings among parents faced with uncertain NBS results, ${ }^{14,18,19}$ less is known about how health-care providers navigate this challenge. Although over $75 \%$ of physicians report that they would openly communicate uncertain diagnostic results with patients, ${ }^{20}$ many clinicians do not act on this instinct in practice if they believe that patients will react negatively. ${ }^{21}$ Additionally, some clinicians may respond to uncertainty with potentially unnecessary testing, referrals, and treatments. ${ }^{21}$ Other providers choose a more passive monitoring approach to avoid lengthy diagnostic odysseys and potentially invasive treatments for their patients. ${ }^{22}$ In general, uniform algorithms and guidelines help clinicians navigate uncertainty. However, there is no pan-Canadian equivalent to the Recommended Uniform Screening Panel (RUSP), a harmonized panel of target conditions in place to standardize screening programs and minimize jurisdictional discrepancies in the United States. Screening panels in Canada are determined by provincial programs, resulting in wide variation across provinces. In an emerging era of genotype- first NBS, it is critical to understand how providers perceive NBS-generated uncertainty to optimize screening algorithms and postscreening care. This study aims to describe how metabolic care providers navigate medical uncertainty when managing IMDs, using nonclassic forms of primary NBS targets as illustrative examples.

\section{MATERIALS AND METHODS}

\section{Design, setting, and participants}

Following an exploratory qualitative design and in partnership with the Canadian Inherited Metabolic Disease Research Network (CIMDRN), ${ }^{23}$ health-care providers involved in caring for children with IMD in 1 of 14 metabolic care centers in Canada were invited to participate in semistructured interviews. The 14 centers are supported by seven NBS programs in Canada. Between February and April 2018, 34 CIMDRN-affiliated metabolic physicians and 15 dietitians were invited to participate through an electronic invitation letter sent by an internal CIMDRN correspondent. Following the initial invitation, two reminder emails were sent to minimize nonresponse and snowball sampling was conducted to recruit genetic counselors and nurses from these specialized care centers. This study was approved by the Research Ethics Board at the Hospital for Sick Children (SickKids), in Toronto, Ontario, Canada.

\section{Data collection}

Variant forms of five primary screening targets were selected as illustrative, rather than exhaustive, examples of NBSgenerated uncertainty. Result types selected for discussion included variant medium-chain acyl-CoA dehydrogenase deficiency (v-MCADD), variant very long-chain acyl-CoA dehydrogenase deficiency ( $v$-VLCADD), mild hyperphenylalaninemia (MHP), partial biotinidase deficiency (partial BIO), and 3-methylcrotonyl-CoA carboxylase deficiency (3MCCD). These results present challenges related to diagnostic, prognostic, and therapeutic uncertainty and represented the majority of nonclassic IMD forms in Ontario at the inception of the study. ${ }^{24}$ Table 1 elaborates on the classic and variant forms of each of the selected screening targets.

Data were collected through open-ended, semistructured telephone interviews conducted by one member of the study team (P.J.A.). Informed consent was obtained from all participants. Interview questions asked respondents to reflect 
Table 1 Screening targets and associated variants

\begin{tabular}{|c|c|c|c|}
\hline Classic form & Description & Laboratory results & Variant form \\
\hline Phenylketonuria (PKU) & $\begin{array}{l}\text { Deficiency of phenylalanine hydroxylase, } \\
\text { an enzyme that converts phenylalanine } \\
\text { into tyrosine. Dissimilar from PAH } \\
\text { cofactor (tetrahydrobiopterin, BH4) } \\
\text { deficiency. Phenylalanine (Phe) } \\
\text { accumulates and can cause permanent } \\
\text { intellectual disability. Treated with Phe- } \\
\text { free diet. }\end{array}$ & $\begin{array}{l}\text { Phenylalanine }>600 \mu \mathrm{mol} / \mathrm{l} \text { on dried } \\
\text { bloodspot (DBS). High phenylalanine: } \\
\text { tyrosine ratio. } \mathrm{BH} 4 \text { deficiencies } \\
\text { excluded on DBS. Molecular genetic } \\
\text { testing is not essential but may help } \\
\text { determine residual activity and severity } \\
\text { of phenotype. } 1101 \text { known PAH } \\
\text { variants. }\end{array}$ & $\begin{array}{l}\text { Mild hyperphenylalaninemia (MHP): } \\
\text { elevated Phe below diagnostic } \\
\text { threshold for PKU. Diagnostic } \\
\text { uncertainty due lack of universal } \\
\text { threshold. Therapeutic uncertainty } \\
\text { caused by lack of consensus and little } \\
\text { evidence for Phe-free diet. }\end{array}$ \\
\hline $\begin{array}{l}\text { Profound biotinidase } \\
\text { deficiency }\end{array}$ & $\begin{array}{l}\text { A neurocutaneous disorder caused by } \\
\text { insufficient endogenous biotin } \\
\text { production. This can cause seizure, } \\
\text { respiratory problems, developmental } \\
\text { delay, ataxia, eczema, hearing loss, vision } \\
\text { problems, and alopecia. Treated with } \\
\text { biotin supplements. }\end{array}$ & $\begin{array}{l}\text { Low enzyme activity measured from } \\
\text { DBS. }<10 \% \text { of activity on confirmatory } \\
\text { serum test. Molecular genetic testing } \\
\text { for biallelic pathogenic BTD variants } \\
\text { may resolve ambiguous } \\
\text { enzymatic tests. }\end{array}$ & $\begin{array}{l}\text { Partial biotinidase deficiency: } \\
10-30 \% \text { serum biotinidase activity. } \\
\text { Prognostic uncertainty whether natural } \\
\text { history is symptomatic. Lower dose } \\
\text { biotin supplementation is generally } \\
\text { recommended. }\end{array}$ \\
\hline
\end{tabular}

NBS newborn screening.

on their direct personal experience managing the uncertainty associated with each of the selected result types. The interviewer then explored patient and family communication strategies in the context of uncertainty, and perceptions of the clinical and policy relevance of NBS-generated uncertainty. The interview guide was iteratively revised to elicit more descriptive detail, probing specific clinical experiences in greater depth. Example questions are provided in Table 2. Data were collected until the point of saturation, where new insights ceased to emerge. ${ }^{25}$

\section{Data analysis}

Data analysis was performed according to the principles of qualitative description. ${ }^{25}$ Audio files were transcribed verbatim. Interview transcripts were reviewed and marginal notes were recorded to develop an intuition of emerging patterns. Two researchers (P.J.A. and R.Z.H.) reviewed initial transcripts and developed a preliminary coding scheme. Early codes were established deductively, guided by the Han et al. ${ }^{17,26} 3$-dimensional taxonomy. In later stages, the analysis shifted toward a more inductive coding approach, as the taxonomy-driven deductive approach was not able to fully capture salient themes. Using qualitative analytic software (NVivo 11) as an organizing tool, one researcher (P.J.A.) applied the codeveloped codebook (P.J.A.). A second team member (R.Z.H.) read a subsample of transcripts to verify or refute the coding structure and coding decisions. Coding discrepancies were resolved through discussion. 
Table 2 Example interview questions

\begin{tabular}{|c|c|}
\hline Example question & $\begin{array}{l}\text { Concept mapping to 3D } \\
\text { taxonomy }\end{array}$ \\
\hline $\begin{array}{l}\text { Reflecting on your diversity of } \\
\text { experiences, can you walk me through } \\
\text { the process of interpreting uncertain lab } \\
\text { results pertaining to [variant disorder]? }\end{array}$ & $\begin{array}{l}\text { Scientific issue: diagnostic } \\
\text { aspects of uncertainty }\end{array}$ \\
\hline $\begin{array}{l}\text { From your perspective, what } \\
\text { comparisons do you observe between } \\
\text { how families react when their child is } \\
\text { initially diagnosed with [variant disorder] } \\
\text { versus [classic form of disorder]? }\end{array}$ & $\begin{array}{l}\text { Personal issue: psychological } \\
\text { aspects of uncertainty }\end{array}$ \\
\hline $\begin{array}{l}\text { How do you think [variant disorder] } \\
\text { should be managed and how does this } \\
\text { compare to clinically available practice } \\
\text { guidelines, algorithms, and protocols? }\end{array}$ & $\begin{array}{l}\text { Scientific issue: therapeutic } \\
\text { aspects of uncertainty }\end{array}$ \\
\hline $\begin{array}{l}\text { What strategies would you recommend } \\
\text { to other health-care providers if they } \\
\text { have to communicate an uncertain result } \\
\text { to a family in the context of [variant } \\
\text { disorder]? }\end{array}$ & $\begin{array}{l}\text { Personal issue: social aspects } \\
\text { of uncertainty }\end{array}$ \\
\hline $\begin{array}{l}\text { Some might say that there is a lack of } \\
\text { clear direction and consensus in } \\
\text { managing [variant disorder]. How are } \\
\text { local protocols or care plans developed } \\
\text { at your institution for managing this } \\
\text { disorder? }\end{array}$ & $\begin{array}{l}\text { Practical issue: procedural } \\
\text { aspects of uncertainty }\end{array}$ \\
\hline
\end{tabular}

\section{RESULTS}

The study sample consisted of 12 health-care providers specialized in IMD care across seven Canadian provinces. Participants were recruited from nine specialized, tertiary care, metabolic centers in urban settings that serve large geographic areas. In total, 65 providers were invited to participate, with no response from 53 invitees. All responding providers, including metabolic physicians, biochemical geneticists, nurses, and genetic counselors, were interviewed, including seven female respondents and five male respondents. Although some participants assumed multiple professional roles (e.g., program directors, university professors, and researchers), their dominant role was health-care provider. Interview duration was 30 to 60 minutes.

Three themes were identified. The first theme was developed deductively through the application of the 3dimensional taxonomy. ${ }^{26}$ Participants described diagnostic, prognostic, therapeutic, and personal issues of uncertainty in the context of selected metabolic disorders (Fig. 2). Inductively, participants described the importance of erring on the side of caution, advocating for prescriptive management strategies to prevent adverse outcomes. In addition, they highlighted the importance and effectiveness of clear communication and consistency when navigating medical uncertainty.

\section{Theme 1: categories of uncertainty in newborn screening} Based on personal experience with caring for children with the selected IMDs, providers described various issues and sources of uncertainty. Drawing on their general experience with screening, participants suggested "it was not unusual to get a finding that wasn't clearly pathologic or clearly benign and therefore, led to uncertainty" (P3). Diagnostic issues of uncertainty arose from indeterminate results due to an out-ofrange laboratory finding or a genetic variant associated with a nonclassic IMD form. In the context of MCADD and VLCADD, one provider remarked, "the concern really lies [in] if we're able to properly make a diagnosis...normal or not significantly elevated [results] put a lot of doubt in your mind whether the newborn screening is just showing carrier status or benign biochemical abnormalities, so we may pursue genetic testing; sometimes we try to do enzyme testing, which requires skin biopsies. It can be quite invasive for a little baby" (P8, MCADD/VLCADD). Reflecting diagnostic uncertainty in the context of MCADD and VLCADD, another respondent said, "we wonder about...the possibility that the baby's actually a heterozygote...that can create some uncertainty with respect to how to manage the patients... and contributes to [a] longer period for diagnosis" (P5). In the context of confirmatory MHP testing, a provider remarked, "starting with whether or not to do a biopterin loading test in the neonatal period-there is a fair bit of variation there. Whether or not to do genotyping is another place where you see variation" (P10).

Therapeutic issues of uncertainty also arise. For MCADD and VLCADD, one provider said, "I'm not sure what the risk of sudden decompensation is and I'm not sure to what degree to aggressively intervene in intercurrent illness" (P10). Clinicians cited a need for "clear guidelines about who needs treatment [and to] what degree they need treatment," and wondered, "are we overtreating some of these patients and taking undue or unnecessary precautions?" (P5, MCADD/ VLCADD). Pertaining to MCADD, another provider said, "I put one or two kids on carnitine and you know I have a colleague who is on the side of never starting carnitine and we got into quite the argument" (P10). For MHP, a respondent commented, "between 360 and $600[\mu \mathrm{mol} / \mathrm{L}$ Phe] is questionable whether you treat...some would consider sapropterin [if their confirmatory test shows responsiveness] but because it's so expensive and not always covered...I'm not sure that's something that should be routinely done" (P3, MHP). Therapeutic uncertainty may benefit some stakeholders; "of course, the drug company would argue that nondietary treatment would be the way to go" (P10, MHP). Nonharmful, cost-effective treatments result in less therapeutic uncertainty. For partial BIO, for example, a participant reflected, "I think we're probably overtreating a lot with pharmacologic doses of biotin, but it's a pretty innocuous treatment" (P7, BIO). Comparing complete and partial BIO, a health-care provider said, "the evidence is less clear, but we typically tend to advise treatment with biotin supplementation [for partial BIO] because it's cheap, it's safe, it's effective, 

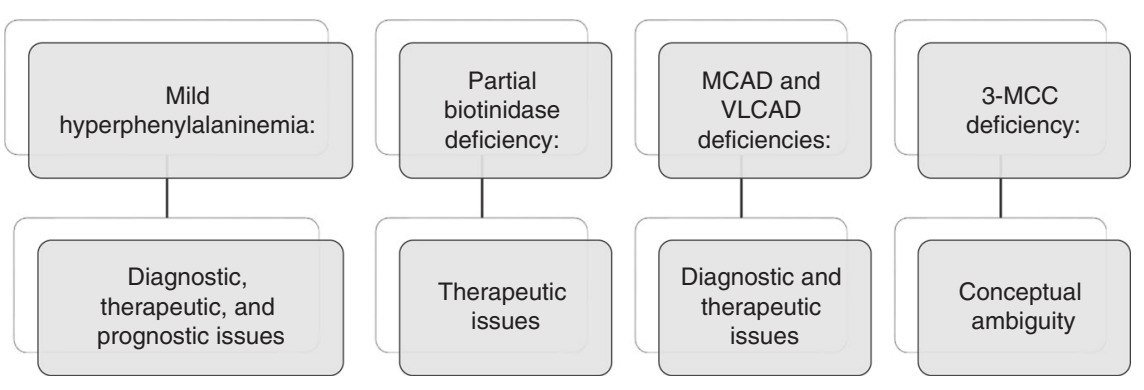

Fig. 2 Variant disorders and categories of uncertainty. 3-MCC 3-methylcrotonyl-CoA carboxylase, MCAD medium-chain acyl CoA dehydrogenase, $V L C A D$ very long-chain acyl COA dehydrogenase.

and it could potentially eliminate the risk of a complication" (P5).

Prognostic issues of uncertainty were also discussed. For example, one participant said, "we don't want to overmedicalize a condition that is benign...we still want to follow them [but] not as frequently as someone with classic PKU" (P12). Participants identified prognostic ambiguity as a source of uncertainty; 3-MCCD was described as a "so-called disease" (P1), a "real/not real disease" (P2), a "nondisease" (P3, P10), a "benign condition" (P4, P8), and a "nil pathological condition" (P10). Another provider stated, "there may be the occasional patient, very occasional, who has a disease associated with 3-MCC, and there's some controversy, if one speaks to different people, about whether it should or shouldn't be included" (P3). One clinician said, "we dealt with criticism from the American Organic Acidemia Association... there's people at some centers who really do believe that this is a disease [anticipating an adverse prognosis]... and treat their kids much more aggressively" (P10, 3-MCCD).

Providers identified personal issues of uncertainty at the locus of their patients. Referring to NBS generally, a respondent remarked, "it would be very disconcerting to hear about the baby having an abnormal newborn screen when the family...doesn't understand what you're talking about and didn't even know their baby had a newborn screen" (P7). Another respondent said, "if we're screening for a condition and there's...no evidence to guide decisions about treatment or no treatment...then you could potentially argue that screening for it is causing more harm because you're causing...distress to families and subjecting them to testing and appointments" (P5, 3-MCCD). Other sources (i.e., probability and complexity) and issues (i.e., causal explanations and practical) of uncertainty from the 3-dimensional taxonomy were not represented in the data.

\section{Theme 2: providers tend to err on the side of caution}

Health-care providers described the importance of medical caution in their approach to managing screen-positive patients. In general, one participant said, "to prevent morbidity and mortality in their child...we are going to do things that err on the side of caution, to keep the baby safe" (P2). Participants associated caution with a prescriptive approach to navigating uncertainty. One participant said, "if we know that this child is at, perhaps, increased risk, we don't want to take chances and this is why we usually overtreat those individuals. You don't want to take the chance that after 10 years you learn one of your mild cases-where you have said 'no risk'-has passed away" (P11, MCADD/VLCADD). Another specialist said, "cautious means, erring on the side of overtreating...you're overemphasizing the benefits of treatment and underemphasizing the potential harms of treatment in that situation" (P4, 3-MCCD). One respondent tells parents who receive indeterminate results, "you're going to have to keep an eye on your baby and make sure that he or she doesn't do this list of things that we say is concerning" (P2). Providers indicate that this may result in stress for parents: "we put in that increased anxiety for them to always be watching their baby" (P8, MCADD/VLCADD). One respondent stated that, in general, "we have to be careful, because we don't consider it completely harmless to treat a child as affected...there are potential harms associated with hospitalizing kids during minor illnesses" and added "dietary changes are not necessarily benign interventions" (P5).

\section{Theme 3: clear and consistent parent-provider communication is needed}

Providers emphasized the need to be clear when communicating with families and the importance of developing consensus within and across specialized metabolic care centers. One provider said that, in general, "it can get disorienting, because we think, 'Oh, did I do that last time? Or the time before?' or, 'How often should I be doing this?' and...'Why am I poking this child? Am I actually going to change things based on what I do? Is it going to make a difference?"” (P2). Another respondent said, "being open about the evidence and the existence of medical uncertainty doesn't mean...I'm presenting things with less confidence... I'll say, 'The approach here is permissive...let's make a decision together" (P10). In the words of one participant, "there's a little bit of an art to metabolic care, it's not all strict science" (P2). Without reference to a specific disorder, another respondent said, "I feel that being very honest with patients, or families-telling them what we think-being honest when we don't know...I feel that these are all key things to build up a good relationship and I feel that very often it helps to manage uncertainty and people's anxiety" (P12). The absence of a pan-Canadian screening strategy left some clinics struggling to develop guidelines of their own that 
account for uncertainty. One participant remarked, "I think there's been a slide towards being more aggressive in treating [MHP patients] because the American recommendations are pushing that" (P4). Specialists "reach out to other clinics across the country and ask, what are you doing with your [MHP patients]?" (P1). However, some participants suggested that a communication gap could be filled at a national level. One participant said, "hopefully they can find a [way to implement] united screening, country-wide...that would be my goal" (P9). Other providers said, "there is not a lot of communication between the different newborn screening programs" (P12) and "I think it's important that not every province has to reinvent the wheel every time they want to add new things to their newborn screen" (P1).

\section{DISCUSSION}

This study presents the first pan-Canadian narrative related to how health-care providers navigate uncertainty generated by newborn screening for IMD. First, the results show that providers have concerns about the diagnostic, therapeutic, prognostic, and personal issues associated with uncertainty, which persist, resolve, or are amplified by confirmatory testing. Second, although guidelines provide clear recommendations for managing classic cases, this work shows that providers favor erring on the side of caution for managing variant and nonclassic cases. Third, participants indicated that open dialogue with patients and a consistent approach to case management are effective strategies where uncertainty exists. Although prior studies indicate that clinicians are hesitant to describe personal uncertainties, ${ }^{27,28}$ all providers in this study openly described clinical challenges and circumstances arising from uncertainty. Our findings align with prior research, suggesting uncertainty may increase ordering of diagnostic tests and result in diagnoses that may be assigned prematurely. $^{20-22}$

Respondents indicated that balance is needed between discontinuing surveillance to prevent medicalization and erring on the side of caution to prevent adverse health outcomes. These findings parallel physician responses to uncertain prognoses in the context of neonatal hypoxic-ischemic encephalopathy, in which a respondent remarked, "if the prognosis is quite uncertain probably the tendency would be to treat, to continue care, and to counsel accordingly." ${ }^{29}$ Brennan et al. ${ }^{30}$ define medicalization as "the redefining or reconceptualizing of nonmedical behaviors, experiences, or problems as medical in nature." For example, the safe target value for serum phenylalanine has been lowered since the 1960s, driving an increase in the proportion of hyperphenylalaninemia patients requiring ongoing follow up. $^{31,32}$ Treatment of MHP through a phenylalanine-free diet and monitoring may prevent intellectual disability but also carries lifelong social and physical costs. ${ }^{33}$ Medication-based approaches (sapropterin dihydrochloride) may cost families up to US $\$ 129,800$ per annum in this population. ${ }^{34}$ Providers' expressed preference to treat may be driven by physician discomfort with uncertainty, fear of unfavorable outcomes, or litigation. $^{29}$ However, treating seemingly healthy cases warrants careful consideration of the practical and psychosocial impacts on children and families placed in a "liminal space" between sickness and health. ${ }^{13}$

In the context of the findings reported herein, Han et al.'s ${ }^{26}$ 3-dimensional taxonomy was a partially transferable framework. ${ }^{17}$ Probability and complexity, as sources of uncertainty, were not applicable to participant responses. From the healthcare provider perspective, it seems that scientific issues of uncertainty-specifically diagnostic, prognostic, and therapeutic issues - are of the greatest relevance. Practical issues are likely more relevant from a programmatic or laboratory perspective and personal issues of uncertainty appear to be most relevant to patients. Han et al. ${ }^{26}$ write that "scientific uncertainty is disease-centered, while practical and personal uncertainties are system- and patient-centered, respectively." With the application of genotype-first approaches to NBS, Han et al.'s categories that relate to genetic results, such as pathogenicity of variants, genotype-phenotype association, and pleiotropy, will become increasingly salient. Other frameworks, such as a clinical uncertainty framework developed by Djulbegovic et al. ${ }^{35}$ are generalizable (e.g., “uncertainty about eliciting patients' values, preferences and risk attitudes"), but trade depth for breadth. Han and Djulbegovic $^{36}$ are calling on the genomics community to endorse the 3-dimensional taxonomy as a unifying starting point for inquiry, recognizing the limitations of any model that attempts to address the inherent challenges of categorizing uncertainty. Researchers and clinicians can help refine the taxonomy through an interactive website (https://research. nhgri.nih.gov/taxonomy/). ${ }^{36}$

From a programmatic and policy perspective, respondents in this study believe that screening programs are likely to benefit from reciprocal sharing of treatment algorithms and clinical practice guidelines. This highlights the importance of consensus where evidence is insufficient to direct care with reasonable consistency. Respondents also suggested that strengthened networks between centers and consistent algorithms or guidelines for managing cases would help clinicians navigate uncertainty. Provincial cooperation combined with the use of strategic federal spending power may encourage the development of national guidelines, analogous to the RUSP in the United States. Wilson and colleagues ${ }^{37}$ make a compelling argument for harmonization of a panCanadian screening strategy to ensure equitable access to care regardless of geographical location. Beyond nomination and review of target screening disorders, the Secretary's Advisory Committee on Heritable Disorders in Newborns and Children (SACHDNC) has helped to provide recommendations on informed consent in research, timeliness of screening, and improved data quality. The SACHDNC structure may also serve as a forum for advice and consensus building regarding managing NBS-generated uncertainty.

The availability of high-throughput genotype-first screening approaches will likely enable more cost-effective, rapid, and precise diagnoses for some, while simultaneously resulting in 
longer diagnostic periods and results of varying clinical consequence for others. ${ }^{4}$ For example, Touw et al. ${ }^{9}$ found that variants of unknown significance in the $A C A D M$ gene were observed in about $22 \%$ of MCADD cases. Although current tandem mass spectrometry-based methods may reveal secondary findings, such as the identification of asymptomatic 3-MCCD mothers in screen-positive infants, Howard et al. ${ }^{4}$ warn that challenges associated with uncertainty are likely to increase with nontargeted, genome-wide strategies. There will always be uncertain differences in expressivity due to pleiotropy, incomplete or reduced penetrance, and epigenetic regulation. Characterization of mild and variant phenotypes will likely improve with further research, but uncertainty must be addressed more specifically and transparently by advisory committees and within the scientific and clinical communities.

\section{Limitations}

This study used qualitative description to develop an in-depth account of how uncertainty affects post-NBS care. This approach allowed us to identify a diversity of perspectives across health-care provider types and jurisdictions to illuminate the range of experiences and strategies that exist. Transferability may be limited by the narrow scope of the project, which focused on the clinical management of rare disorders from the perspective of highly specialized clinicians in metabolic care centers. Retrospective interviewing techniques are subject to confirmation and recall bias. Further studies could enhance the reproducibility and transferability of our findings from other perspectives. Thus, further qualitative research is needed to explore both family and programmatic insights on post-NBS care for variant forms of IMD.

\section{Conclusion}

This study found that metabolic health-care providers have concerns about the diagnostic, therapeutic, and prognostic challenges associated with uncertainty, which persist despite confirmatory follow-up testing. Providers also prefer that management strategies for variant forms of screening targets err on the side of caution. In addition, participants indicate that open dialogue with patients and a consistent approach to case management are effective strategies where uncertainty exists. It is hoped that this exploratory work will catalyze future research including the development and trial of information resources for families and providers. The SACHDNC is one forum to develop best practices and recommendations related to the screening, reporting, and medical surveillance of uncertain results in NBS. Given providers' tendency to err on the side of caution, NBS programs can anticipate heightened system utilization, with good intentions, where uncertainty exists. If uncertainty is to be generated with more frequency by shifting technologies, additional responsibilities will be placed on programs and clinical communities to incorporate the consequences of uncertainty into the benefit-risk calculus of NBS.

\section{ACKNOWLEDGEMENTS}

The researchers acknowledge the support of the University of Toronto and The Hospital for Sick Children Centre for Genetic Medicine for their sponsorship of this work. The researchers also thank coinvestigators Jonathan Kronick, Fiona A. Miller, John Mitchell, Andreas Schulze, Cheryl Greenberg, Aziz Mhanni, Sylvia Stockler, Hilary Vallance, and Karen Tam, as well as the Canadian Inherited Metabolic Diseases Registry and Network for their support. This work was funded by the SickKids Centre for Genetic Medicine Catalyst Grant.

\section{DISCLOSURE}

The authors declare no conflicts of interest.

Publisher's note Springer Nature remains neutral with regard to jurisdictional claims in published maps and institutional affiliations.

\section{REFERENCES}

1. Centers for Disease Control and Prevention. Ten great public health achievements-United States, 2001-2010. MMWR Morb Mortal Wkly Rep. 2011;60:619-623.

2. Currier RJ, Sciortino S, Liu R, Bishop T, Koupaei RA, Feuchtbaum L. Genomic sequencing in cystic fibrosis newborn screening: what works best, two-tier predefined CFTR mutation panels or second-tier CFTR panel followed by third-tier sequencing? Genet Med. 2017;19:1159.

3. Ceyhan-Birsoy O, Murry JB, Machini K, et al. Interpretation of genomic sequencing results in healthy and ill newborns: results from the BabySeq Project. Am J Hum Genet. 2019;104:76-93.

4. Howard HC, Knoppers BM, Cornel MC, et al. Whole-genome sequencing in newborn screening? A statement on the continued importance of targeted approaches in newborn screening programmes. Eur J Hum Genet. 2015;23:1593-1600.

5. Botkin JR, Clayton EW, Fost NC, et al. Newborn screening technology: proceed with caution. Pediatrics. 2006;117:1793-1799.

6. Jay AM, Conway RL, Feldman GL, Nahhas F, Spencer L, Wolf B. Outcomes of individuals with profound and partial biotinidase deficiency ascertained by newborn screening in Michigan over 25 years. Genet Med. 2015;17:205-209.

7. Niu D-M, Chien Y-H, Chiang C-C, et al. Nationwide survey of extended newborn screening by tandem mass spectrometry in Taiwan. J Inherit Metab Dis. 2010;33:295-305.

8. Maier EM, Liebl B, Röschinger W, et al. Population spectrum of ACADM genotypes correlated to biochemical phenotypes in newborn screening for medium-chain acyl-CoA dehydrogenase deficiency. Hum Mutat. 2005;25:443-452.

9. Touw C, Smit G, de Vries M, de Klerk J, Bosch A, Berry G. Risk stratification by residual enzyme activity after newborn screening for medium-chain acyl-CoA dehyrogenase deficiency: data from a cohort study. Orphanet J Rare Dis. 2012;7:1-8.

10. van Wegberg AMJ, MacDonald A, Ahring K, et al. The complete European guidelines on phenylketonuria: diagnosis and treatment. Orphanet J Rare Dis. 2017;12:162.

11. Arnold GL, Van Hove J, Freedenberg D, et al. A Delphi clinical practice protocol for the management of very long chain acyl-CoA dehydrogenase deficiency. Mol Genet Metab. 2009;96:85-90.

12. Wilcken B. 3-Methylcrotonyl-CoA carboxylase deficiency: to screen or not to screen? J Inherit Metab Dis. 2016;39:171-172.

13. Timmermans S, Buchbinder M. Patients-in-waiting: living between sickness and health in the genomics era. J Health Soc Behav. 2010;51: 408-423.

14. Perobelli S, Zanolla L, Tamanini A, Rizzotti P, Assael BM, Castellani C. Inconclusive cystic fibrosis neonatal screening results: long-term psychosocial effects on parents. Acta Paediatr. 2009;98:1927-1934.

15. Massie J, Gillam L. Uncertain diagnosis after newborn screening for cystic fibrosis: an ethics-based approach to a clinical dilemma. Pediatr Pulmonol. 2014;49:1-7. 
16. Green RC, Goddard KA, Jarvik GP, et al. Clinical sequencing exploratory research consortium: accelerating evidence-based practice of genomic medicine. Am J Hum Genet. 2016;98:1051-1066.

17. Han PKJ, Umstead KL, Bernhardt BA, et al. A taxonomy of medical uncertainties in clinical genome sequencing. Genet Med. 2017;19:918.

18. Tluczek A, McKechnie AC, Lynam PA. Modified uncertainty theory and parents' perspectives about equivocal diagnostic results for cystic fibrosis. Qual Health Res. 2010;20:209-223.

19. Grob R. Is my sick child healthy? Is my healthy child sick?: changing parental experiences of cystic fibrosis in the age of expanded newborn screening. Soc Sci Med. 2008;67:1056-1064.

20. Gerrity M, Earp J, Devellis R, Light D. Uncertainty and professional work: perceptions of physicians in clinical practice. Am J Sociol. 1992;97: 1022-1051.

21. Alam R, Cheraghi-Sohi S, Panagioti M, Esmail A, Campbell S, Panagopoulou E. Managing diagnostic uncertainty in primary care: a systematic critical review. BMC Fam Pract. 2017;18:79.

22. Almond SC, Summerton N. Test of time. BMJ. 2009;338:b1878.

23. Potter BK, Chakraborty P, Kronick JB, et al. Achieving the "triple aim" for inborn errors of metabolism: a review of challenges to outcomes research and presentation of a new practice-based evidence framework. Genet Med. 2013;15:415.

24. Annual report to the Newborn Screening Ontario Advisory Councilcalendar year 2017. 2017. https://www.newbornscreening.on.ca/sites/ default/files/2017_nso_annual_report_final_public.pdf. Accessed January 2018.

25. Bradshaw C, Atkinson S, Doody O. Employing a qualitative description approach in health-care research. Glob Qual Nurs Res. 2017;4:233339 3617742282.

26. Han PKJ, Klein WMP, Arora NK. Varieties of uncertainty in health-care: a conceptual taxonomy. Med Decis Making. 2011;31:828-838.

27. Schneider A, Wubken M, Linde K, Buhner M. Communicating and dealing with uncertainty in general practice: the association with neuroticism. PLOS ONE. 2014;9:e102780.

28. Seaburn DB, Morse D, McDaniel SH, Beckman H, Silberman J, Epstein R. Physician responses to ambiguous patient symptoms. J Gen Intern Med. 2005;20:525-530.

29. Rasmussen LA, Cascio MA, Ferrand A, Shevell M, Racine E. The complexity of physicians' understanding and management of prognostic uncertainty in neonatal hypoxic-ischemic encephalopathy. J Perinatol. 2019;39:278-285.

30. Brennan R, Eagle L, Rice D. Medicalization and marketing. J Macromarketing. 2010;30:8-22.
31. van Spronsen F. Mild hyperphenylalaninemia: to treat or not to treat. J Inherit Metab Dis. 2011;34:651-656.

32. Ohlsson A, Bruhn $H$, Nordenström A, Zetterström RH, Wedell A, von Döbeln $U$. The spectrum of PAH mutations and increase of milder forms of phenylketonuria in Sweden during 1965-2014. JIMD Rep. 2016;34:19-26.

33. van Wegberg AMJ, MacDonald A, Ahring $K$, et al. The complete European guidelines on phenylketonuria: diagnosis and treatment. Orphanet J Rare Dis. 2017;12:162.

34. Canadian Agency for Drugs and Technologies in Health (CADTH). Common Drug Review: Pharmacoeconomic Review Report on sapropterin dihydrochloride (Kuvan). https://www.cadth.ca/sites/default/ files/cdr/pharmacoeconomic/SR0472_Kuvan_PE_Report_e.pdf. Accessed July 2018 .

35. Djulbegovic B, Hozo I, Greenland S. Uncertainty in clinical medicine. In: Gifford S, Gabbay D, Thagard P, Woods J (eds.) Handbook of the philosophy of science. Vol. 16. Philosophy of medicine. Elsevier; 2011; Chapter 10, pp. 299-356.

36. Han PKJ, Djulbegovic B. Tolerating uncertainty about conceptual models of uncertainty in health-care. J Eval Clin Pract. 2019;25: 183-185.

37. Wilson K, Kennedy S, Potter BK, Garaghty M, Chakraborty P. Developing a national newborn screening strategy for Canada. Health Law Rev. 2010;18:31-39.

Open Access This article is licensed under a Creative Commons cc) Attribution-NonCommercial-NoDerivatives 4.0 International License, which permits any non-commercial use, sharing, distribution and reproduction in any medium or format, as long as you give appropriate credit to the original author(s) and the source, and provide a link to the Creative Commons license. You do not have permission under this license to share adapted material derived from this article or parts of it. The images or other third party material in this article are included in the article's Creative Commons license, unless indicated otherwise in a credit line to the material. If material is not included in the article's Creative Commons license and your intended use is not permitted by statutory regulation or exceeds the permitted use, you will need to obtain permission directly from the copyright holder. To view a copy of this license, visit http://creativecommons.org/licenses/by-nc-nd/4.0/.

(C) The Author(s) 2019 